\title{
Quantum Cellular Automata: a Short Overview of Molecular Problem
}

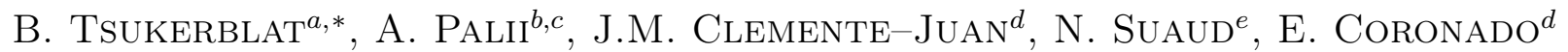 \\ ${ }^{a}$ Ben-Gurion University of the Negev, Beer-Sheva, Israel \\ ${ }^{b}$ Institute of Problems of Chemical Physics, Chernogolovka, Russia \\ ${ }^{c}$ Institute of Applied Physics, Academy of Sciences of Moldova, Kishinev, Moldova \\ ${ }^{d}$ Instituto de Ciencia Molecular, Universidad de Valencia, Paterna, Spain \\ ${ }^{e}$ Laboratoire de Chimie et Physique Quantiques, Université de Toulouse, Toulouse, France
}

\begin{abstract}
In this article we summarize the study of the electronic states and electron-vibrational interactions in squareplanar molecular entities playing the role of molecular cells in quantum cellular automata (QCA), a promising paradigm of quantum computing. The following issues are shortly discussed: 1) QCA as a paradigm of quantum computing; 2) molecular implementation of $\mathrm{QCA}$; 3) vibronic coupling as the origin of charge trapping, encoding of binary information 4) non-linear cell-cell response; 4) spin-switching in molecular QCA based on mixed-valence cells; 5) multimode dynamic vibronic problem, the symmetry assisted approach.
\end{abstract}

DOI: 10.12693/APhysPolA.133.329

PACS/topics: 75.50.Xx; 87.16.A-

\section{Introduction}

As predicted by the "Moore law" the conventional computer chips double their density approximately every two years. That is why the quantum computing became a challenging way in the development in this area. The most serious obstacle in the realization of quantum computing is the decoherence which should be sufficiently long or, at least, not shorter than duration of the gate logical operation. As was demonstrated [1, 2]), one could reach a decoherence time in a molecular magnet that would permit quantum information processing. A perspective route to proceed in this fundamental problem exploits paradigm of QCA in which quantum dots or molecules play role of the carriers of the binary information (see Refs [2-12]). The use of QCA allows to create a new kind of computing "Bypassing the transistor paradigm" (see Lent's article [8] with this indicative title). The proposal gave a strong impact to the development of the new multidisciplinary field of nanotechnology that combines physics, chemistry, material science, computer science, and electrical engineering. The QCA devices promise to consume extremely small amounts of power ensuring at the same a very small heat release and high switching speed.

\section{The main idea of quantum cellular automata}

The cell (Fig. 1) consists of the four quantum dots or redox sites in a mixed valence (MV) molecule occupied by two extra electrons which can tunnel between the sites inside the cell but cannot tunnel between different cells. The information is encoded in the two antipodal charge configurations of the cell and transferred via

\footnotetext{
*corresponding author; e-mail: tsuker@bgu.ac.il
}

the Coulomb interaction between the neighboring cells as schematically shown in Fig. 1. The two charge configurations are separated by a potential barrier. These quasi-stable charge configurations are able carry binary information (binary $\mathbf{0}$ or $\mathbf{1}$ ) which is encoded in the cell polarization.

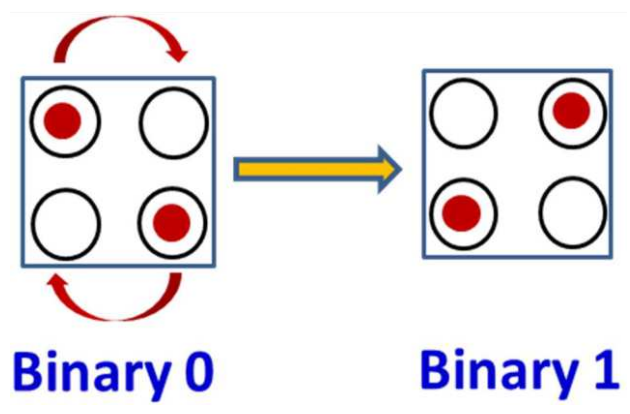

Fig. 1. Charge distributions in a four-dot quantum cell or in a tetrameric MV molecule encoding binary $\mathbf{0}$ and 1 via the charge distributions; bi-electronic jump from binary $\mathbf{0}$ to $\mathbf{1}$.

\section{Quantum cellular automata: molecular implementation}

The idea of using molecular systems as QCA cells instead of semiconductor dots has been proposed and discussed [9]. Molecular QCA have significant advantages, like possibility to create ultra-high device densities operating at room temperature, providing at the same time options to control the key properties of the molecule by chemical means. As single molecule implementation of $\mathrm{QCA}$, it was proposed [9] to use 1,4-dialyl butane radical cation consisting of butyl bridge linking two allyl groups accommodating electron. Among attractive candidates for molecular cells [9] MV molecules [12, 13] are especially promising because they have two or more sites available 
for extra electrons forming specific charge configurations while the electron-lattice interaction can create the barrier between different charge distributions. This refers to a variety of MV systems studied in molecular magnetism, such as metal dimers and mainly nanoscopic MV systems, like reduced polyoxometalates (e.g., Keggin and Wells-Dawson systems) accommodating two or several electrons delocalized over a metal network [14-16].

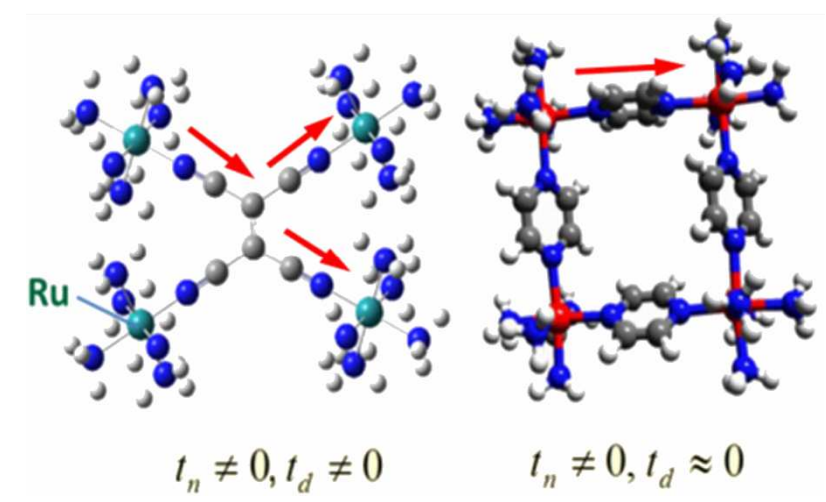

Fig. 2. Molecular QCA cells: two tetra-ruthenium complexes exhibiting different electron networks of the transfer pathways (denoted by red arrows) for the electronic pair [17].

As examples the two MV tetra-ruthenium complexes which are suitable for molecular implementation of the QCA can be mentioned [12, 17] (Fig. 2). A great potentiality of molecules as the leading candidates to be implemented in QCA is based on the following substantial advantages: (a) as distinguished from the quantum dot cells, molecular cells of specified composition are identical and therefore have identical physical characteristics; (b) they can be engineered via chemical tailoring to have desired controllable characteristics; (c) molecular cells are relatively large (but still nanoscale!) objects, much larger than ions and, thus, accessible for individual addressing while processing the information; (d) such molecules can be attached to different types of platforms by grafting clusters on solid surfaces.

\section{Electronic spectrum of a mixed-valence molecular cell}

To treat the bi-electronic MV four site system we introduce the Hamiltonian involving three terms describing the main relevant interactions:

$$
H=H_{e}+\sum_{i} \hbar \omega_{i}\left(Q_{i}^{2}-\frac{\partial^{2}}{\partial Q_{i}^{2}}\right)+V .
$$

Here $H_{e}$ is the Hubbard Hamiltonian, including all interactions between electrons and ions. The next two terms describe the free molecular vibrations and the electronvibrational interaction $V$. The Hamiltonian $H_{e}$ includes the following components [18, 19]: (1) Coulomb repulsion between the two itinerant electrons which depends on whether these electrons are instantly localized at the adjacent sites (along the sides of the square) or they occupy the remote sites (antipodal positions). The Coulomb repelling forces tend to keep the electrons as far as possible in order to minimize the energy, i.e. on the sites located at opposite vertices. The difference in the energies of the Coulomb repulsion between the distant pairs $U_{d}$ and the neighboring ones $U_{d}$ ( $d$ - and $n$-pairs having two and four configurations respectively) will be denoted as $U$ (Fig. 3). The charge configurations in the two lowest states are assumed to encode the binary information in molecular QCA. (2) The transfer of the two electrons among the four sites. The electron transfer parameters $t_{n}$ and $t_{d}$ (Fig. 2) correspond to the jumps between the distant and adjacent sites respectively. The direct magnetic exchange coupling will be neglected.

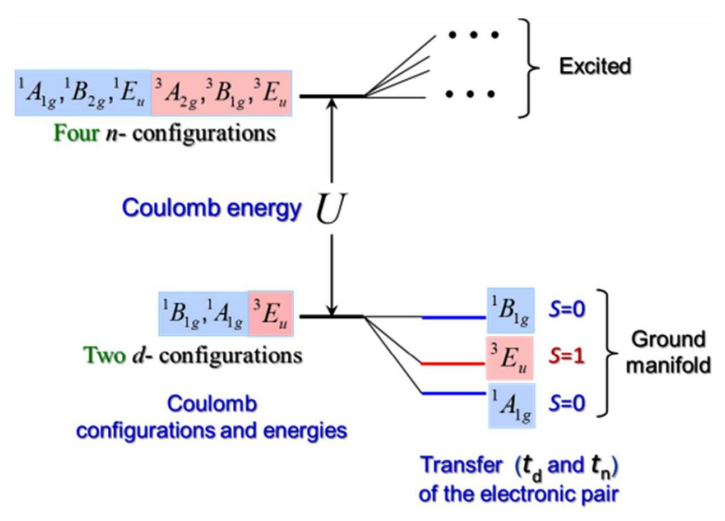

Fig. 3. General view of the energy pattern created by the Coulomb interactions and transfer processes in a tetrameric bi-electronic molecular unit. For the sake of simplicity the excited states with energies around $U$ are not shown.

The electronic extended Hubbard-type Hamiltonian $H_{e}$ of the cell can be represented as:

$$
\begin{aligned}
& H_{e}=U_{n} \sum_{i} \sum_{\sigma, \sigma^{\prime}} n_{i, \sigma} n_{i+1, \sigma^{\prime}}+U_{d} \sum_{i} \sum_{\sigma, \sigma^{\prime}} n_{i, \sigma} n_{i+2, \sigma^{\prime}} \\
& +t_{n} \sum_{i} \sum_{\sigma}\left(a_{i, \sigma}^{+} a_{i+1, \sigma}+a_{i+1, \sigma}^{+} a_{i, \sigma}\right) \\
& +t_{d} \sum_{i} \sum_{\sigma}\left(a_{i, \sigma}^{+} a_{i+2, \sigma}+a_{i+2, \sigma}^{+} a_{i, \sigma}\right)
\end{aligned}
$$

where $a_{i, \sigma}^{+}\left(a_{i, \sigma}\right)$ creates (annihilates) an electron occupying active orbital at the site $i$ with spin projection $\sigma$, $n_{i, \sigma}$ are the occupation number operators. The summation is performed over the pairs of the neighboring sites (1st and 3rd terms) and over the pairs of distant sites (2nd and 4th terms). The first term in Eq. (2) describes the interelectronic Coulomb repulsion $\left(U_{n}\right)$ between the adjacent sites, while the second term $\left(U_{d}\right)$ is that for the remote sites. The intra-site repulsion gives rise to the highly excited levels and therefore is excluded in the present consideration.

The group-theoretical classification of the energy levels gives the following spin-singlet and spin-triplet terms (Fig. 3) for the $d$-type configurations: $\quad{ }^{1} B_{1 g}(d), \quad{ }^{1} A_{1 g}(d), \quad{ }^{3} E_{u}(d)$. For the the $n$-type configurations one finds: 
${ }^{1} B_{2 g}(n),{ }^{1} E_{u}(n),{ }^{1} A_{1 g}(n),{ }^{3} A_{2 g}(n),{ }^{3} B_{1 g}(n),{ }^{3} E_{u}(n)$ as shown in Fig. 3. The explicit expressions for energy levels are given in Ref. [18]. The electron transfer processes in a bi-electron system separate spin-triplets and spin-singlets, resulting thus in an effective magnetic interaction between the electrons. This kind of the effective magnetic exchange caused by electron delocalization has been recognized in our earlier consideration of MV double reduced polyoxometales with Keggin and Wells-Dawson structures, which allowed us to explain the unusual diamagnetism of these compounds [20, 21]. The microscopic estimations were done in Ref. [22].

\section{Jahn-Teller vibronic model of molecular cell}

The vibronic interaction in MV system [23-25] is known to produce bistability which leads to a barrier between the two charge distributions being thus one of the key factors in the QCA. We will use the vibronic model formulated by Piepho, Krausz and Schatz (PKS model) [23]. The model operates with the "breathing" displacements of the ions around the sites which are assumed to be independent. This model is rather simple but it properly reflects the main features of the vibronic coupling in MV systems, in particular, the presence of the potential barrier separating localized configurations. The PKS coordinates in a tetrameric system span the irreps $A_{1 g}, B_{1 g}, E_{u}$ of the point group $\boldsymbol{D}_{4 h}$. One can obtain the following expressions for the PKS coordinates for a square-planar tetrameric system belonging to this point group:

$$
\begin{aligned}
Q_{B_{1 g}} & =\frac{1}{2}\left(Q_{A}-Q_{B}+Q_{C}-Q_{D}\right), \\
Q_{E_{u} x} & =\frac{1}{\sqrt{2}}\left(Q_{A}-Q_{C}\right), \\
Q_{E_{u} y} & =\frac{1}{\sqrt{2}}\left(Q_{B}-Q_{D}\right) .
\end{aligned}
$$

In Eqs. (3) $Q_{\alpha}$ are the PKS coordinates composed of the four "breathing" vibrations $Q_{i}$ associated with the sites $(i=A, B, C, D)$ as schematically shown in Fig. 4 (the full-symmetric coordinate is not shown). In the case of the MV system under consideration, the linear vibronic interaction can be represented in the matrix form as follows:

$$
V=\sum_{\alpha} v_{\alpha} \boldsymbol{O}_{\alpha} Q_{\alpha}
$$

where the values $v_{\alpha}$ are the vibronic parameters and the matrices $\boldsymbol{O}_{\alpha}$ are given in Ref. [18]. In the PKS model the frequencies and the coupling parameters $v\left(v_{\alpha} \equiv v\right)$ prove to be equal for all active vibrations. Taking into account the symmetry of the electronic states, one can see that we are dealing with the combined Jahn-Teller (JT) and pseudo JT vibronic problems $\left({ }^{1} B_{1 g}+2^{1} A_{1 g}+{ }^{1} B_{2 g}+{ }^{1} E_{u}\right) \otimes\left(b_{1 g}+e_{u}\right)$ for spinsinglets and $\left({ }^{3} A_{2 g}+{ }^{3} B_{1 g}+2^{3} E_{u}\right) \otimes\left(b_{1 g}+e_{u}\right)$ for spintriplets terms. One can see that the JT and pseudo JT effects are the inherent ingredient of the description of the quantum cell in QCA.

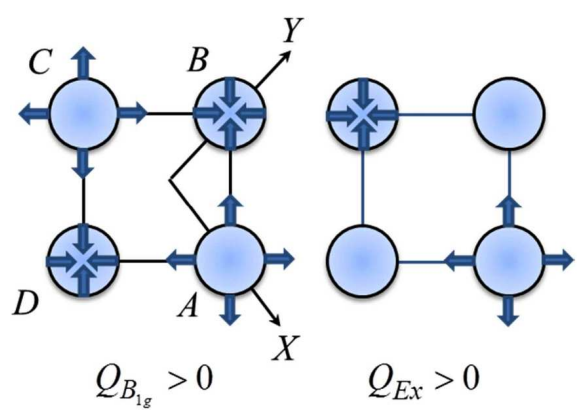

Fig. 4. Pictorial representation of the symmetry adapted vibrational PKS coordinates of a square-planar unit. Only $x$-component of the double degenerate $E$ type vibration is shown.

\section{Vibronic localization in bi-electronic molecular cell}

Since the binary information is encoded in the antipodal distribution of the electrons the Coulomb repulsion should considerably exceed the transfer integrals in order to ensure the maximal distance between electrons. Provided that the condition of strong Coulomb repulsion $U \gg\left|t_{n}\right|,\left|t_{d}\right|$ is fulfilled, the low lying group of levels ( ${ }^{1} B_{1 g},{ }_{1} A_{1 g},{ }^{3} E_{u}$ ) is well isolated (Fig. 3) and therefore the vibronic problem can be restricted to the ground manifold being thus reduced to one-mode $\left({ }^{1} B_{1 g}+{ }^{1} A_{1 g}\right) \otimes b_{1 g}$ and ${ }^{3} E_{u} \otimes b_{1 g}$ subtasks [18]. This means that a pair of the levels with $S=0\left({ }^{1} B_{1 g},{ }^{1} A_{1 g}\right)$ are mixed through the only vibration $b_{1 g}$ and the same vibration is active in the $S=1$ term ${ }^{3} E_{u}$. One can see that in the framework of the PKS model the $e$-modes do not act within the ground manifold because they mix the ground Coulomb configurations of $d$-type with excited $n$ type configurations. The vibronic pseudo JT problem proves to be one-dimensional and the adiabatic potentials are found as:

$$
\begin{aligned}
& \varepsilon_{ \pm}(Q)=-\frac{\Delta}{2}+\frac{\hbar \omega}{2} Q^{2} \pm \frac{1}{2} \sqrt{\Delta^{2}+4 v^{2} Q^{2}} \\
& \varepsilon_{x, y}(Q)=-\Delta_{1}+\frac{\hbar \omega}{2} Q^{2} \pm v Q .
\end{aligned}
$$

Here the following notations are used: $8 t_{n}^{2} / U$ $16 t_{n}^{2} t_{d} / U^{2}-\Delta_{1}$. In Eq. (5) the functions $\varepsilon_{ \pm}(Q)$ are the two branches of the adiabatic potential for the $\left({ }^{1} B_{1 g}+{ }^{1} A_{1 g}\right) \otimes b_{1 g}$ pseudo JT problem, while $\varepsilon_{x, y}(Q)$ are the surfaces for the static JT problem ${ }^{3} E_{u} \otimes b_{1 g}$. Depending on the interrelation between the gap $\Delta$ and the strength of the vibronic coupling $v$, the lower sheet $\varepsilon_{-}(Q)$ can have a single minimum (Fig. 5) at $Q_{+}=0$, providing weak coupling (or/and large gap $\Delta), v^{2}<\Delta \hbar \omega / 2$, or two minima at the points $Q_{ \pm}=$ $\pm \sqrt{v^{2} / \hbar^{2} \omega^{2}-\Delta^{2} / 4 v^{2}}$ under the condition of strong coupling (or/and small gap $\Delta$ ), $v^{2}>\Delta \hbar \omega / 2$ (Fig. 6). The energy of the single minimum of $\varepsilon_{-}(Q)$ in which the electronic pair is fully delocalized is just the electronic energy $(-\Delta)$. The potential curves $\varepsilon_{x, y}(Q)$ represent two intersecting parabolas and exhibit two minima irrespec- 


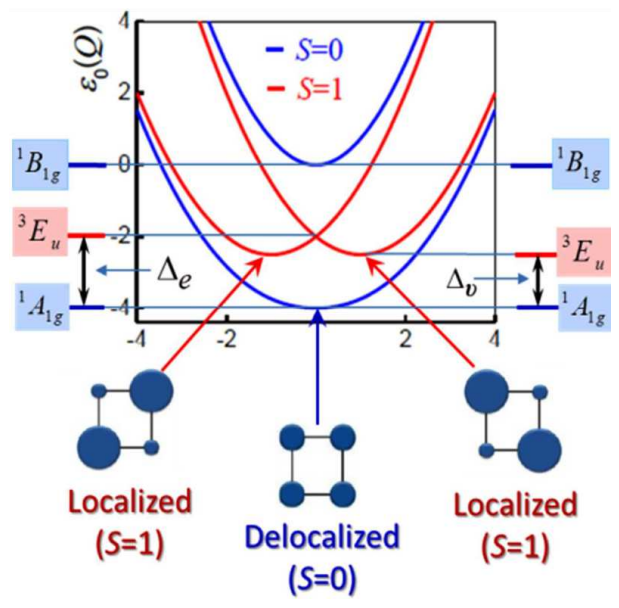

Fig. 5. Adiabatic potentials of a square planar MV unit for the low lying levels belonging to $d$ configurations of the electronic pair. The case of weak vibronic coupling.
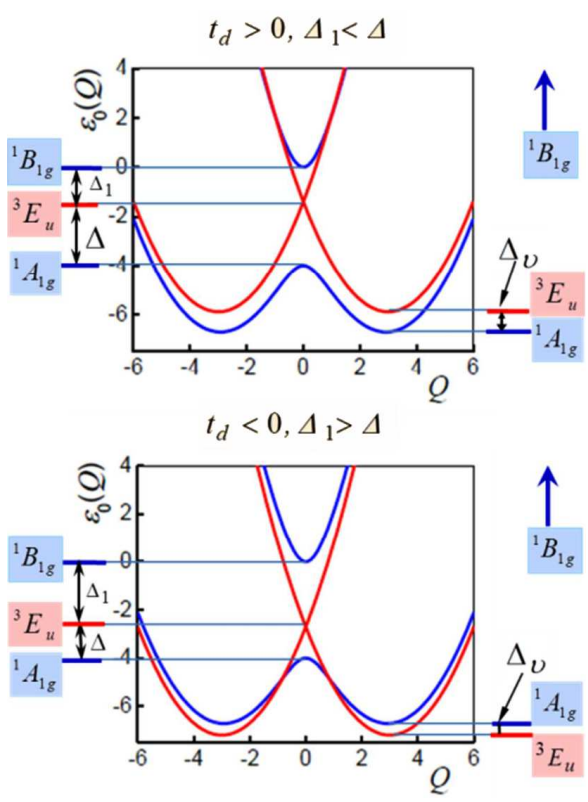

Fig. 6. Adiabatic potentials of a square planar MV unit for the low lying levels belonging to $d$ configurations of the electronic pair. The case of strong vibronic coupling. $\quad \varepsilon_{0}(Q)=\varepsilon(Q) / \hbar \omega$-dimensionless adiabatic energy, $v_{0}=v / \hbar \omega, \Delta / \hbar \omega=4$. Red - spin triplets, blue — spin-singlets.

tively of the value of the vibronic coupling (static JT effect) which are disposed at $Q_{x}=-Q_{y}=v / \hbar \omega$ and have the energies $-\Delta_{1}-v^{2} / 2 \hbar \omega$. These two minima correspond to the localization of the electronic pair in the antipodal positions ("broken symmetry" states). In the case of static JT effect, the tunneling between the minima is forbidden and so the electronic pair is fully localized in each of the two minima irrespectively of the strength of the vibronic coupling. To distinguish differ- ent physical situations in Fig. 6 we use the dimensionless parameter $\kappa=\left(2 \Delta_{1}-\Delta\right) / \Delta_{1}$, which is expressed through $t_{d}$ as $\kappa=4 t_{d} / U$ and serves as a measure of the position of the spin-triplet ${ }^{3} E_{e}$ within the gap between two spin-singlets ${ }^{1} B_{1 g}$ and ${ }^{1} A_{1 g}$ in the electronic spectrum. Thus, providing $\kappa=0$ the spin-triplet is located just in the middle of this gap. One can see that the vibronic coupling effectively reduces the singlet-triplet gap $\left({ }^{1} A_{1 g},{ }^{3} E_{u}\right)$ in both cases, weak and strong coupling as shown in Figs. 5 and 6 . In the limit of strong coupling and providing $\kappa=0$, the minima for the spin-singlet and spin-triplet states become practically degenerate. These minima correspond to the full localization of the charges along the diagonals of the square for both spin configurations. This is a result of the full quenching of the $t_{n}$ processes by a strong vibronic interaction, which leads to the localization of the electronic pair and, consequently, to the paramagnetic ground state. Providing $t_{d}<0$, the minima of spin-singlet states are deeper than those for the spin-triplet states although the singlet-triplet gap in the electronic spectrum (at $Q=0$ ) is reduced. In the case of $t_{d}>0$ the strong enough vibronic coupling leads to the spin-crossover of the levels, so that the spin-triplet state becomes the ground one and the system is locked at the minima.

\section{Spin-dependent localization in a mixed-valence cell}

The main efforts in the area of QCA have been focused on the analysis of electronic charge density distribution within the cell and its change due to polarization (electric quadrupole moment) induced by the Coulomb field of the neighboring cells. As one could see from the adiabatic consideration in Sect. 6, there is a strong correlation between the molecular spin and the degree of localization in mixed valence clusters. In Ref. [19] we focused on the study of spin-effects in a single squareplanar MV cell comprising two electrons and in coupled molecular cells for QCA. Using the vibronic model it was demonstrated that the polarizabilities of the cell are different in spin-singlet and spin-triplet states of the electronic pair. Based on this inference the concept of spinswitching in molecular QCA has been proposed and the conditions under which this effect is feasible have been derived. In order to reveal these conditions we have performed a series of quantum-mechanical calculations of the vibronic energy levels of the isolated cell and of the cell subjected to the external polarizing field. The spinswitching effect stemming from the theoretical model is shown to be dependent on the relative strength of the intra-cell electronic interactions (Coulomb repulsion energies and electron transfer parameters) and the vibronic coupling. This is expected to suggest a route for observation of spin-switching in which the non-magnetic vibronic ground state of the cell is changed to the magnetic one due to polarization induced in this cell by the neighboring one. The spin switching is shown to produce a significant effect on the cell-cell response function leading to the unusual stepwise shape of this function. 


\section{Cell-cell response function within the vibronic model}

We have considered the electronic and vibronic properties of the isolated molecular cells in QCA. Implementation of the logically significant OCA arrays or circuits requires consideration of the "intercommunication" between the molecular cells. It is essential that the charge configuration of the input cell is able to dictate the charge configuration of the output cells. In the simplest case of a QCA wire one has to solve the non-stationary problem of the signal propagation from the input cell in a definite state (binary "1" or " 0 ") to the output. In a QCA fanout circuit an input signal is transformed into two or more output signals. Providing different cellular automata architectures one should assume that the state of a cell should be strongly influenced by the state of the neighboring cell (or cells), so that a slight polarization of a cell would induces nearly complete polarization of a neighboring cell. To characterize this kind of highly nonlinear behavior of a bistable cell the use of the cell-cell response function has been proposed [26]. Since the vibronic coupling produces a trapping effect, it is expected to produce substantial influence on the cell-cell response function. Let us consider the polarization of the cell, a scalar quantity Pintroduced by Lent et al $[3,9]$ as a normalized measure of the degree to which the electron densities are localized in antipodal positions along the diagonals $A C$ or $B D$ of a square planar four-site cell:

$$
P=\frac{\left(\rho_{A}+\rho_{C}\right)-\left(\rho_{B}+\rho_{D}\right)}{\rho_{A}+\rho_{C}+\rho_{B}+\rho_{D}},
$$

where $\rho_{i}$ are the electronic charges on the sites. If the sites $B$ and $D$ are empty $\left(\rho_{B}=\rho_{D}=0, \rho_{A}=\rho_{C}=1\right)$ the cell is fully polarized along $A C$ and so $P=-1$. Alternatively, if the electrons occupy sites $B$ and $D$, polarization $P=+1$. From the qualitative arguments one can conclude that in MV systems the effective electronic charges are the results of the competitive effects of the vibronic (JT and pseudo JT) coupling and transfer processes. Due to completion of these two interactions the system is localized only partially so that the electronic charges are distributed among the sites as schematically shown in Fig. 7. One can introduce the values $U_{A C}$ and $U_{B D}$ which are the Coulomb interaction energies of cell 1 in the two antipodal localizations of the electronic pair with the cell 2 having a certain polarization $P_{2}$. The electronic densities for the linear configuration (Fig. 6) of the input and output cells are calculated in Ref. [18] within the quantum-mechanical (dynamical) approach to the vibronic problem.

The vibronic coupling strongly modifies the cell-cell response function. From Fig. 8 one can observe that the increase of the vibronic coupling parameter leads to the increase of the abruptness of the $P_{1}$ vs $P_{2}$ curves, while at smaller values of $v$ the steepness of the curve is less pronounced, and even at $P_{2}= \pm 1$ the polarization $\left|P_{1}\right|$ remains smaller than 1 . This is evidently due to the fact that the vibronic coupling tends to localize the electrons
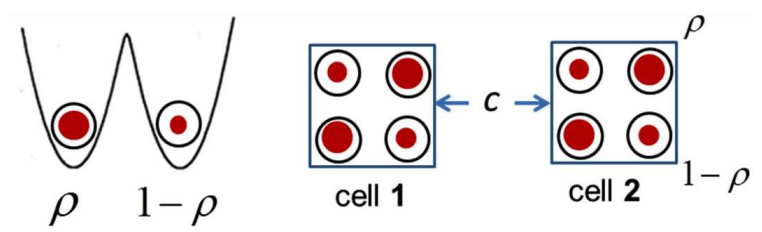

Fig. 7. Schematic picture of the partial delocalization at the minima of a double-well adiabatic potential (left) and Coulombic cell-cell interaction resulting in the alignment of the two cells (right.

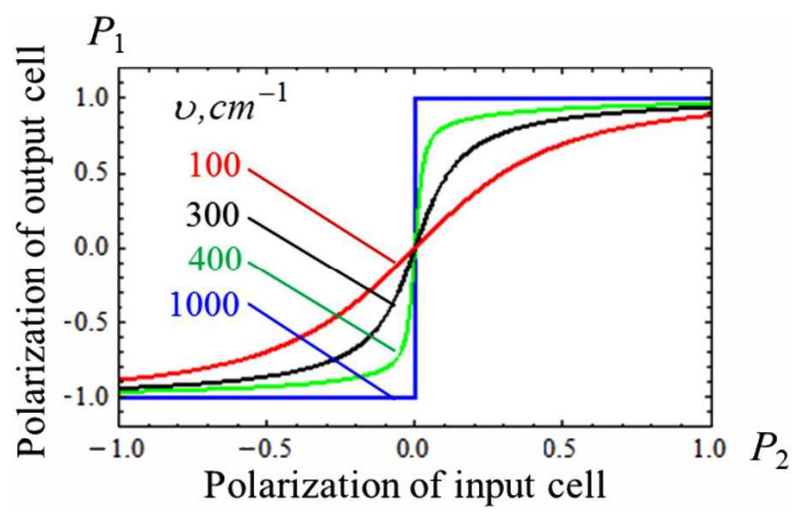

Fig. 8. Influence the vibronic pseudo JT coupling on the cell-cell response function.

(reducing their mobility) and, therefore, it decreases polarization of the cell. More detailed consideration, in particular, estimation of the parameters for some MV clusters is given in Ref. [18].

Finally, we should mention a new concept in molecular QCA which has not been discussed here for the sake of brevity. We proposed to employ $\left[\mathrm{V}_{8}^{\mathrm{IV}} \mathrm{V}_{4}^{\mathrm{V}} \mathrm{As}_{8} \mathrm{O}_{40}\left(\mathrm{H}_{2} \mathrm{O}\right)\right]^{4+}$ polyoxometalate as a quantum inverter. The detailed quantum-chemical study shows that this proposal is feasible.

\section{Non-adiabatic approach}

to the vibronic problem of molecular cell

The adiabatic approximation (Born-Oppenheimer paradigm) is valid in the cases of strong JT vibronic coupling for the low lying levels of the systems. But the adiabatic approximation loses its accuracy providing moderate vibronic coupling. Moreover, by definition, this approximation (based on classical representation of the ionic movement) fails in the description of the discrete vibronic spectrum and, in particular, in the evaluation of the intervalence optical bands in MV systems. By the definition the adiabatic description assumes that the nuclear (ligand) motion is much slower than the electronic one. This is, in general, invalid near the anticrossing area of the adiabatic surfaces. In fact, Fig. 9 illustrates the non-adiabatic coupling in this area caused by the fast Landau-Zener transitions. This physical argument is common for the all kinds of vibronic systems. 


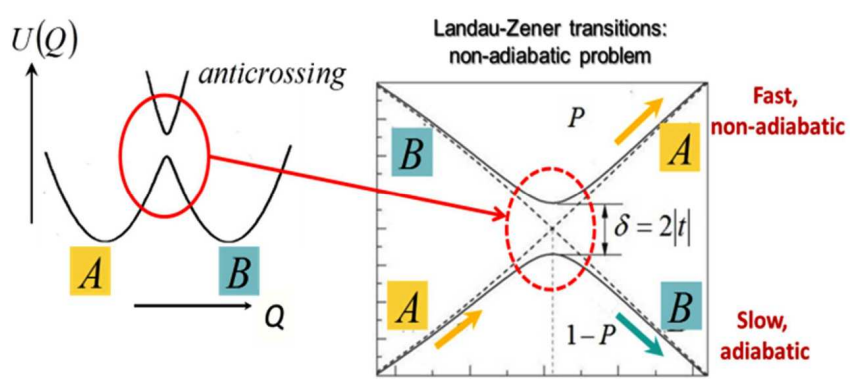

Fig. 9. Illustration for the non-adiabatic coupling in a MV dimer with a double-well potential: avoided level crossing and Landau-Zener transitions.

Understanding of limitations of the BornOppenheimer approximation gave rise to a new trend at the borderline between physics and chemistry that can be referred to as "Chemical theory beyond the Born-Oppenheimer paradigm" (see recent book [27]). Alternatively, the quantum-mechanical approach usually leads to a complicated (often practically unsolvable) dynamic problem in multimode systems, especially in cases when several electronic states are coupled to the JT and pseudo-JT modes (see review in [28, 29]). The computational difficulties originate from the high dimensionalities of the excited vibrational levels of the harmonic vibrations. This occurs, for example, in the nanoscopic MV systems, which can be exemplified by polyoxometalates [15]. Therefore, a precise solution of the quantum-mechanical vibronic problem is of primary importance for the evaluation of the shapes of the electron transfer optical absorption bands and quantitative analysis of the main parameters of tetrameric quantum cells.

To solve the multidimensional JT problem we have recently suggested [30-32] a theoretical tool which has been referred to as "symmetry assisted approach" which takes advantage from the point symmetry of the system. The symmetry assisted approach allows to block the vibronic matrices to full extent in accordance with the point symmetry of the systems and thus to make tractable the complicated dynamic problems for these molecular cells. The mathematical details of the approach are described in Refs. [30-32]. The main idea is that the evaluation of the vibrational functions can be performed through the action of the symmetry adapted polynomial operators $\boldsymbol{T}_{\Gamma \gamma}^{(n)}$ onto the vacuum state:

$$
\boldsymbol{T}_{\Gamma \gamma}^{(n)}\left|\forall n_{i}=0\right\rangle=|n, \Gamma \gamma\rangle,
$$

where $\Gamma$ is the vibronic irrep and $\gamma$ numerates the basis. In this way one realizes the transformation from the basis of the simple products of the harmonic oscillator functions $\left|n_{1}, n_{2}, \ldots n_{k}\right\rangle$ with a certain $n$ (a definite vibrational level, $n=n_{1}+n_{2}+\ldots n_{k}$ ) to the symmetry adapted basis $|n, \Gamma \gamma\rangle$ :

$$
|n, \Gamma \gamma\rangle=\sum_{\left\{. . n_{i} . .\right\}} c\left(. . n_{i} . ., n, \nu \Gamma \gamma\right)\left|. . n_{i} . .\right\rangle,
$$

where $\nu$ is an additional quantum number which is in- troduced in order to distinguish the states of the system when the irrep $\Gamma$ occurs several times in the set with a definite $n$. For this reason $\boldsymbol{T}_{\Gamma \gamma}^{(n)}$ are referred to as multivibronic symmetry adapted creation operators that can be considered as the complex irreducible tensor operators of the type of $\Gamma \gamma$ in the actual point group $\left(\mathbf{D}_{4 h}\right.$ in the case under consideration). This allows one to build the multivibronic operators with the use of the well known technique for manipulation with the irreducible tensor operators in the point groups:

$$
\boldsymbol{T}_{\Gamma \gamma}^{(n)}=\left\{\left\{\left\{c_{\Gamma_{1}}^{+} \otimes c_{\Gamma_{2}}^{+}\right\}_{\Gamma_{12}} \otimes c_{\Gamma_{3}}^{+}\right\}_{\Gamma_{123}} \ldots \otimes c_{\Gamma_{n}}^{+}\right\}_{\Gamma \gamma},
$$

where $\left\{c_{\Gamma_{1}}^{+} \otimes c_{\Gamma_{2}}^{+}\right\}_{\Gamma_{12} \gamma}$ is component $\gamma$ of the tensor product of the type of $\Gamma \gamma$ the irreducible tensor operators $c_{\Gamma_{1} \gamma_{1}}^{+}$and $c_{\Gamma_{2} \gamma_{2}}^{+}$. The operators $\boldsymbol{T}_{\Gamma \gamma}^{(n)}$ are built up by the successive coupling of the creation operators corresponding to all active vibrations. Then the symmetry adapted vibrational functions are coupled to the electronic ones to get the full basis. The vibronic matrix is blocked according to the irreducible representations of the vibronic functions which significantly reduces the Hilbert space.

The calculations are implemented in a specially designed software (which will be published elsewhere). With this tool we also extended the theory of the molecular QCA cell beyond the limit of prevailing Coulomb repulsion in the electronic pair (as described in previous sections). Consequently the general pseudo JT problems for spin-singlet $\left({ }^{1} B_{1 g}, 2^{1} A_{1 g},{ }^{1} B_{2 g},{ }^{1} E_{u}\right) \otimes\left(b_{1 g}+e_{u}\right)$ and spin-triplet states $\left({ }^{3} A_{2 g},{ }^{3} B_{1 g}, 2^{3} E_{u}\right) \otimes\left(b_{1 g}+e_{u}\right)$ in a square- planar bi-electronic system has been recently solved [32]. The obtained symmetry adapted electronvibrational functions have been employed for the calculation of the profiles (shape- functions) of the charge transfer absorption bands in the tetrameric MV complexes and for the discussion of the magnetic properties. In Ref. [32] we presented also the characteristics of the performance of the evaluation which can be referred to as "index of efficiency" defined as the ratio (CPU time for blocked matrix) / (CPU time for full matrix). In particular, use of the symmetry adapted basis leads to a significant reduction of the CPU time, for example for $N=24$ (number of the unperturbed vibrational levels) the CPU time with the use of symmetry is only $7.1 \%$ of the total time without application of the symmetry assisted approach.

\section{Conclusion}

In this short review we have summarized some recent results of the molecular problem of QCA. The main attention was focused on the two problems underlying the molecular QCA implementation: electronic spectrum of the molecular cell and vibronic coupling. The electronic spectrum is treated with the framerk of the Hubbard Hamiltonian. The main conclusions about the low lying levels are made provided that the inter-cell Coulomb repusion exceeds the transfer integrals.

A special attention is paid to the role of the JT and pseudo-JT effects in charge trapping, encoding of binary 
information in the quantum cell and non-linear cell-cell response. We have shortly discussed the spin-switching effect in QCA. The symmetry assisted approach to the dynamic multimode/multilevel JT problem is described. The method allows to efficiently solve the quantummechanical problem of the energy pattern of nanoscopic vibronic systems. The results of recent studies in the area show that the non-adiabatic theory is very efficient at nanoscale and useful for the study of molecular units and electron-vibrational processes in molecular electronics. One can express also an optimistic expectation that the developed vibronic approach could be applicable in general challenging problem of molecular information processing.

\section{Acknowledgments}

The work has been performed with financial support of the Federal Agency of Scientific Organizations (State registration N 0089-2014-0026). E.C. and JM.C-J thank the EU (ERC Advanced Grant SPINMOL), the Spanish MINECO (grants MAT2014-56143-R, CTQ2014-52758$\mathrm{P}$ and Excellence Unit Maria de Maeztu MDM-20150538), and the Generalidad Valenciana (Prometeo and ISIC Programmes of excellence). All authors are grateful to COST, Action CA15128 "Molecular Spintronics (MOLSPIN)".

B.T. thanks the University of Valencia for the Sabbatical Grant (Estades Temporals Investigadors Convidats 2017).

\section{References}

[1] S. Bertaina, S. Gambarelli, T. Mitra, B. Tsukerblat, A. Müller, B. Barbara, Nature 453, 203 (2008).

[2] R.E.P. Winpenny, Angew. Chem. Int. Ed. 47, 2 (2008).

[3] C.S. Lent, P. Tougaw, W. Porod, G. Bernstein, Nanotechnology 4, 49 (1993).

[4] W. Porod, S.G. Lent, G. Bernstein, A.O. Orlov, I. Amlani, G.L. Snider, J.L. Merz, Int. J. Electronics 86, 549 (1999).

[5] E.P. Blair, S.A. Corcelli, S.G. Lent, J. Chem. Phys. 145, 014307 (2016).

[6] P.D. Tougaw, C.S. Lent, J. Appl. Phys. 75, 1818 (1994).

[7] G. Töth, C.S. Lent, J. Appl. Phys.85, 2977 (1999).

[8] C.S. Lent, Science 288, 1597 (2000).

[9] C.S. Lent, B. Isaksen, M. Lieberman, J. Am. Chem. Soc. 125, 1056 (2003).

[10] C.S. Lent, P. Tougaw, W. Porod, Appl. Phys. Lett. 62, 714 (1993).

[11] C.S. Lent, B. Isaksen, IEEE Trans. Electron. Devices 50, 1890 (2003).
[12] M. Lieberman, S. Chellamma, B. Varughese, Y. Wang, S. G. Lent, G. H. Bernstein, G. Snider, F. C. Peiris, Ann. N.Y. Acad. Sci. 960, 225 (2002).

[13] S.B. Braun-Sand, O. Wiest, J. Phys. Chem. A 107, 285 (2003).

[14] J.M. Clemente-Juan, E. Coronado, Coord. Chem. Rev. 193-195, 361 (1999).

[15] P. Kögerler, B. Tsukerblat, A. Müller, Dalton Trans. 39, 21 (2010).

[16] J.M. Clemente-Juan, E. Coronado, A. Gaita-Arińo, Chem. Soc. Rev. 417464 (2012).

[17] S. Záliš, B. Sarkar, C. Duboc, W. Kaim, Monatsh. Chem. 140, 765 (2009).

[18] B. Tsukerblat, A. Palii, J.M. Clemente-Juan, E. Coronado, J. Chem. Physics 143, 134307 (2015).

[19] A. Palii, B. Tsukerblat, J.M. Clemente-Juan, E. Coronado, J. Phys. Chem. C 120, 16994 (2016).

[20] J.J. Borrás-Almenar, J.M. Clemente-Juan, E. Coronado, B.S. Tsukerblat, Chem. Phys. 195, 1 (1995).

[21] J.J. Borrás-Almenar, J.M. Clemente-Juan, E. Coronado, B.S. Tsukerblat, Chem. Phys. 195, 17 (1995); idem, Chem. Phys. 195, 29 (1995).

[22] N. Suaud, A. Gaita-Ariño, J.M. Clemente-Juan, J. Sánchez-Marín, E. Coronado, J. Am. Chem. Soc. 124, 15134 (2002); C.J. Calzado, J.M. ClementeJuan, E. Coronado, A. Gaita-Ariño, N. Suaud, Inorg. Chem. 47, 5889 (2008).

[23] S.B. Piepho, E.R. Krausz, P.N. Shatz, J. Am. Chem. Soc. 100, 2996 (1978).

[24] B.S. Brunschwig, C. Creutz, N. Sutin, Chem. Soc. Rev. 31, 168 (2002).

[25] S.B. Piepho, J. Am. Chem. Soc. 112, 4197 (1990).

[26] C.S. Lent, P.D. Tougaw, J. Appl. Phys. 74, 6227 (1993).

[27] K. Takatsuka, T. Yonehara, K. Hanasaki, Chemical Theory beyond the Born-Oppenheimer Paradigm: Nonadiabatic Electronic and Nuclear Dynamics in Chemical Reactions, World Scientific Publishing, Singapore 2015.

[28] I.B. Bersuker, V.Z. Polinger, Vibronic Interactions in Molecules and Crystals, Springer, Berlin, 1989.

[29] I.B. Bersuker, The Jahn-Teller Effect, Cambridge University Press, 2006.

[30] B. Tsukerblat, A. Palii, J.M. Clemente-Juan, E. Coronado, in : Progress in Theoretical Chemistry and Physics, Vol. 23, Vibronic Interactions and the Jahn-Teller Effect, Eds: M. Atanasov, C. Daul, P.L.W. Tregenna-Piggott, Springer, Berlin, 2011, p. 39.

[31] B. Tsukerblat, A. Palii, J.M. Clemente-Juan, A. Gaita-Arińo, E. Coronado, Int. J. Quantum Chem. 112, 2957 (2012).

[32] J.M. Clemente-Juan, A. Palii, E. Coronado, B. Tsukerblat, J. Chem. Theory \& Comp. 12, 3545 (2016). 